\title{
Investigation of field and diffusion time dependence of the diffusion-weighted signal at ultrahigh magnetic fields
}

\author{
Nicolas Kunz ${ }^{a, b}$, Stéphane V. Sizonenkoa , Petra S. Hüppia , \\ Rolf Gruetter ${ }^{b, c, d}$ and Yohan van de Looij ${ }^{a, b *}$
}

\begin{abstract}
Over the last decade, there has been a significant increase in the number of high-magnetic-field MRI magnets. However, the exact effect of a high magnetic field strength $\left(B_{0}\right)$ on diffusion-weighted MR signals is not yet fully understood. The goal of this study was to investigate the influence of different high magnetic field strengths (9.4 $\mathrm{T}$ and $14.1 \mathrm{~T})$ and diffusion times $(9,11,13,15,17$ and $24 \mathrm{~ms})$ on the diffusion-weighted signal in rat brain white matter. At a short diffusion time $(9 \mathrm{~ms})$, fractional anisotropy values were found to be lower at $14.1 \mathrm{~T}$ than at $9.4 \mathrm{~T}$, but this difference disappeared at longer diffusion times. A simple two-pool model was used to explain these findings. The model describes the white matter as a first hindered compartment (often associated with the extraaxonal space), characterized by a faster orthogonal diffusion and a lower fractional anisotropy, and a second restricted compartment (often associated with the intra-axonal space), characterized by a slower orthogonal diffusion (i.e. orthogonal to the axon direction) and a higher fractional anisotropy. Apparent $T_{2}$ relaxation time measurements of the hindered and restricted pools were performed. The shortening of the pseudo- $T_{2}$ value from the restricted compartment with $B_{0}$ is likely to be more pronounced than the apparent $T_{2}$ changes in the hindered compartment. This study suggests that the observed differences in diffusion tensor imaging parameters between the two magnetic field strengths at short diffusion time may be related to differences in the apparent $T_{2}$ values between the pools. Copyright $\odot 2013$ John Wiley \& Sons, Ltd.
\end{abstract}

Keywords: diffusion tensor imaging; biophysical mechanisms of MR diffusion; fractional anisotropy; high magnetic field strength; diffusion time

\section{INTRODUCTION}

Over the last decade, the number of high-magnetic-field magnets has increased as a result of technical progress. Increasing the magnetic field $\left(B_{0}\right)$ leads to an almost linear increase in the magnetization of the sample (1), which can be used to improve the signal-to-noise ratio (SNR) and resolution and/or to shorten the acquisition time. As such, for short-TE MR techniques, such as phase imaging, the signal gain, together with an increased frequency shift distribution, can be exploited to increase the spatial resolution and to reduce the scanning time (2). Additional effects, such as an increase in the spectral resolution, may also be advantageous for MR techniques, such as spectroscopy $(3,4)$. However, when using a higher magnetic field, the apparent transverse relaxation time $T_{2}$ will also be shortened (5). Therefore, the gain in SNR may be attenuated for techniques that use long TEs, such as diffusion imaging, where TE is constrained by the duration of the diffusion gradients. In addition, the magnetic susceptibility effects are also larger at higher fields, which make rapid imaging, a mandatory technique for in vivo studies, more challenging. For imaging modalities, such as diffusion imaging, the gain of ultrahigh $B_{0}$ has therefore not been demonstrated clearly.

Although diffusion tensor imaging (DTI) (6) has been used to study a wide range of white matter pathologies (7-10), many aspects of the origin of the diffusion-weighted signal remain unclear (11). The main difficulty arises from the fact that the diffusion-weighted signal, which is sensitive to the tissue's microstructure, originates from a complicated and heterogeneous environment. The multiple interactions that can occur between water molecules and cell membranes, organelles or macromolecules all affect the diffusion process. Therefore, it is

\footnotetext{
* Correspondence to: Y. van de Looij, Laboratory for Functional and Metabolic Imaging, Ecole Polytechnique Fédérale de Lausanne, CH F1 602 - Station 6, 1015 Lausanne, Switzerland.

E-mail: yohan.vandelooij@epfl.ch

a N. Kunz, S. V. Sizonenko, P. S. Hüppi, Y. van de Looij Division of Child Growth and Development, University of Geneva, Geneva, Switzerland

b N. Kunz, R. Gruetter, Y. van de Looij Laboratory for Functional and Metabolic Imaging, Ecole Polytechnique Fédérale de Lausanne, Lausanne, Switzerland

C R. Gruetter

Department of Radiology, University of Lausanne, Lausanne, Switzerland

d R. Gruetter

Department of Radiology, University of Geneva, Geneva, Switzerland
}

Abbreviations used: $A D C$, apparent diffusion coefficient: $B_{0}$ main magnetic field strength; CHARMED, composite hindered and restricted model of diffusion; $D_{\perp}$, radial diffusivity; $D_{/ /,}$axial diffusivity; DTI, diffusion tensor imaging; $F A$, fractional anisotropy; $R O I$, region of interest; $S N R$, signal-to-noise ratio; $T_{1}$, longitudinal relaxation time; $T_{2}$, transverse relaxation time; $t_{\text {diff }}$ diffusion time. 
reasonable to believe that, inside a single imaging voxel, multiple water compartments are present which experience different diffusion and MR properties (e.g. diffusivity, orientation, water content and relaxation times).

Several studies have attempted to address this problem by modeling the diffusion-weighted signal in the white matter in different compartments (12-16) with at least two pools: one pool in which water molecules move almost freely and experience hindered diffusion ('Gaussian' diffusion), and the second pool in which water molecules are 'trapped' in a closed compartment and experience restricted diffusion (e.g. diffusion in an impermeable cylinder) (12-16). To be performed, these models require datasets that are acquired with multiple $b$ values and/or diffusion times $\left(t_{\text {diff }}\right)$. Indeed, a change in these acquisition parameters will change the relative weight of these different compartments in the acquired signal. For example, the diffusion time, which corresponds to the period over which the diffusion process is observed, defines the degree of interaction of the water molecules with the surrounding cellular structures. An increase in $t_{\text {diff }}$ will increase the probability that molecules will meet obstacles, and allows the investigation of the different compartment sizes that make up the tissue's microstructure.

This compartmentation of the MR signal is a well-known problem and has already been addressed for $T_{2}$ relaxation time and magnetization transfer studies investigating myelin water content $(17,18)$. Similarly, it has been suggested that the different diffusion compartments also present different apparent $T_{2}$ values (19-22). DTI-derived parameters measured in the same cellular structure and with the same acquisition parameters, but at two different magnetic fields, may thus not be equal as a result of noncorrelated changes in $T_{2}$ as a function of $B_{0}$, between the two pools. Moreover, diffusion barriers, such as cell membranes, generate susceptibility-induced local magnetic fields, which are also dependent on $B_{0}$. Therefore, it is difficult to fully understand the impact of the magnetic field strength on DTIderived parameters. Indeed, the literature reports inconsistent results. Recent studies (23-25) have reported a significantly higher fractional anisotropy (FA), as well as a lower apparent diffusion coefficient $(A D C)$ and radial diffusivity, at 3.0 $\mathrm{T}$ than at $1.5 \mathrm{~T}$ in brain white matter. However, Polders et al. (26) showed a tendency of FA to decrease from 1.5 to $7 \mathrm{~T}$. Finally, several studies comparing 1.5 T with $3 \mathrm{~T}$ (27) and $3 \mathrm{~T}$ with $7 \mathrm{~T}$ (28) did not show any differences in FA as a function of $B_{0}$.

The variability in DTI-derived parameters as a function of the imaging parameters and MR system characteristics makes a direct comparison between different studies difficult. Therefore, the goal of this study was to investigate the influence of the magnetic field strength as a function of the diffusion time on the diffusion-weighted signal in rat brain white matter. To avoid any bias in the results, such a comparison necessitates two MR scanners that have the same characteristics apart from the differing magnetic field strength. As such, we designed a multi-field experiment using two ultrahigh-magnetic-field animal systems that had exactly the same characteristics (i.e. same gradient coils, same consoles and same coil geometries) except for the main magnetic fields of 9.4 T and 14.1 T.

\section{MATERIALS AND METHODS}

Experiments were performed on two different magnets (magnetic field strengths/inner-bore diameters of $9.4 \mathrm{~T} / 31 \mathrm{~cm}$ and $14.1 \mathrm{~T} / 26 \mathrm{~cm}$, Varian/Magnex), both connected to a Direct Drive console (Varian Medical Systems, Palo Alto, CA, USA), and equipped with $12-\mathrm{cm}$ gradient coils ( $400 \mathrm{mT} / \mathrm{m}, 120 \mu \mathrm{s})$. Two custom-built quadrature transmit/receive surface radiofrequency coils were used with diameters of 20 and $21 \mathrm{~mm}$ for $9.4 \mathrm{~T}$ and 14.1 T, respectively. At each magnetic field strength, exactly the same protocol was used, following the Geneva School of Medicine Animal Ethics Committee and the Geneva State Veterinary Service guidelines. Rats $\left(n=5\right.$ for each $B_{0}$ ) lay prone, with their heads secured via ear bars, and were continuously anesthetized under a flow of 1.5-2\% isoflurane mixed with oxygen/air. The body temperature was maintained at $37 \pm 0.5^{\circ} \mathrm{C}$ using a thermo-regulated water circulation.

Fast spin-echo images were acquired for reference (field of view, $23 \times 23 \mathrm{~mm}^{2}$; acquisition matrix, $256 \times 128$; TE/TR=60/6000 ms; eight averages; slice thickness, $0.8 \mathrm{~mm}$; echo train length, 16). First- and second-order shims were adjusted using FASTMAP (29), resulting in water linewidths of $16-20 \mathrm{~Hz}$ for a volume of interest of $5 \times 7 \times 7 \mathrm{~mm}^{3}$ centered in the brain.

A semi-adiabatic double-spin-echo sequence, with a four-shot echo-planar readout and application of reversed readout gradients for Nyquist ghost suppression, was used (30) to acquire DTI data. DTI acquisition parameters were as follows: field of view, $23 \times 15 \mathrm{~mm}^{2}$; matrix size, $128 \times 64$ zero-filled to $256 \times 168$; 10 slices of $0.8 \mathrm{~mm}$ thickness in the axial plane; eight averages; TE/TR $=50 / 2000 \mathrm{~ms}$. Diffusion gradients were applied around the first $180^{\circ}$ pulse (duration $\delta=4 \mathrm{~ms}$ ) and along an icosahedral 21-direction diffusion gradient sampling scheme (31). The acquisition time for each DTI dataset was $60 \mathrm{~min}$. Six repeated DTI acquisitions were performed with diffusion times $\left(t_{\text {diff }}=\Delta-\delta / 3\right)$ of $9,11,13,15,17$ and $24 \mathrm{~ms}$, and diffusion gradient intensities $G$ of $31.8,28.6,26.3,24.4,22.9$ and $19.2 \mathrm{G} / \mathrm{cm}$, respectively, resulting in a fixed $b$ value of $1000 \mathrm{~s} \mathrm{~mm}^{-2}$. Data were analyzed using custom-written Matlab (Mathworks, Natick, MA, USA) routines. The $3 \times 3$ matrix [b] was calculated for each diffusion gradient orientation, taking into account both diffusion and imaging gradients (32). Diffusion MRI data were fitted to a tensor model using a linear least-squares fit to the equation In $\left(\left[S_{i}\right] / S_{0}\right)=-\left[b_{i}\right][D]$ on a pixel-by-pixel basis. Following diagonalization of the full diffusion tensor, eigenvalues were derived and diffusivity values, ADC (mean of the eigenvalues: $D_{/ /}$, largest eigenvalue; $D_{\perp}$, mean of the smallest and median eigenvalues) and fractional anisotropy (FA) were computed.

Regions of interest (ROIs) were drawn on the directionencoded color maps in the corpus callosum for 10 consecutive slices of the rat brain, and averaged over two slices to yield five different measurement locations (Fig. 1).

$T_{2}$ measurements in the corpus callosum were performed on four rats for each $B_{0}$. A stimulated echo multi-slice sequence (33) with conventional gradient-echo readout was used. A field of view of $20 \times 10 \mathrm{~mm}^{2}$ on a single 1 -mm-thick sagittal slice centered on the rat brain with a matrix size of $96 \times 64$ zero-filled to $128 \times 128$ was acquired at five different TEs $(18,23,28,38$, $48 \mathrm{~ms}$ at both 9.4 and $14.1 \mathrm{~T}$ ) and TR $=3 \mathrm{~s}$. For each TE, the acquisition time was 13 min.

By applying diffusion gradients perpendicular to the fiber direction and with a long diffusion time, the hindered diffusion-weighted signal should be attenuated significantly, leading to a diffusion-weighted signal mainly coming from the restricted compartment. As such, a second $T_{2}$ measurement was performed with the application of diffusion gradients perpendicular to the corpus callosum fibers (rostro-caudal direction) 


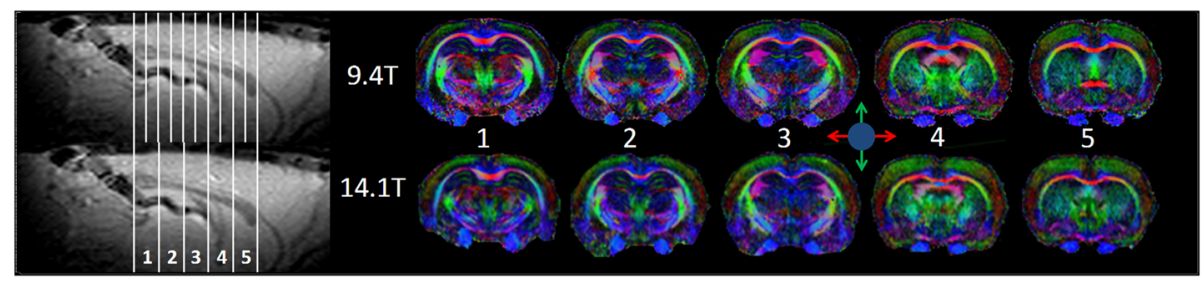

Figure 1. Left: positions of the five axial averaged diffusion tensor imaging (DTI) planes (1-5) overlaid on a $T_{2}$-weighted sagittal image. Right: typical direction-encoded color maps of a rat brain at 9.4 $\mathrm{T}$ (top) and $14.1 \mathrm{~T}$ (bottom) with $t_{\text {diff }}=24 \mathrm{~ms}$. The signal-to-noise ratios (SNRs) calculated on anatomic images $(b=0)$ were $59 \pm 12$ and $57 \pm 16$ for $9.4 \mathrm{~T}$ and $14.1 \mathrm{~T}$, respectively.

with a diffusion time $t_{\text {diff }}$ of $150 \mathrm{~ms}\left(b=4013 \mathrm{~s} \mathrm{~mm}^{-2} ; G=28.4 \mathrm{G} / \mathrm{cm}\right.$; $\delta=2 \mathrm{~ms}$; mixing time, 136.4, 131.4, 126.4, 116.4, $106.4 \mathrm{~ms}$; number of averages, 18, 20, 22, 30, 36; leading to acquisition times of 58, 64, 71, 96 and 116 min for the five TEs, respectively). Data analysis was again performed using routines written inhouse in MATLAB. The corpus callosum was manually delineated and the collected data were fitted with a modified Levenberg-Marquardt algorithm to derive $T_{2}$.

All image SNRs were calculated from the ratio of $\left\langle S_{\text {tissue }}\right\rangle$, the mean signal intensity in a region of the brain (i.e. cortex and/or corpus callosum) obtained from the reference image $\left(b_{0}\right.$ image), relative to $\mathrm{SD}_{\text {noise, }}$ the standard deviation of a large $\mathrm{ROI}$ positioned in the background noise that was visually devoid of artifacts. The collected data at different diffusion times or different image planes for a given $B_{0}$ were submitted to a Friedman nonparametric test (two-way analysis of variance with a multiple comparison of cell medians after a Bonferroni adjustment to compensate for multiple comparisons), and differences between data acquired at 9.4 and 14.1 T were assessed by a Mann-Whitney nonparametric test.

\section{RESULTS}

Overall, the SNR in the superior part of the cortex was equal for the 9.4 $\mathrm{T}(\mathrm{SNR}=59 \pm 12)$ and $14.1 \mathrm{~T}(\mathrm{SNR}=57 \pm 16)$ magnets (Fig. 1).

\section{Regional differences}

In the corpus callosum, several regional differences were observed at both $B_{0}$ (Fig. 2). Mainly, the part that corresponds to
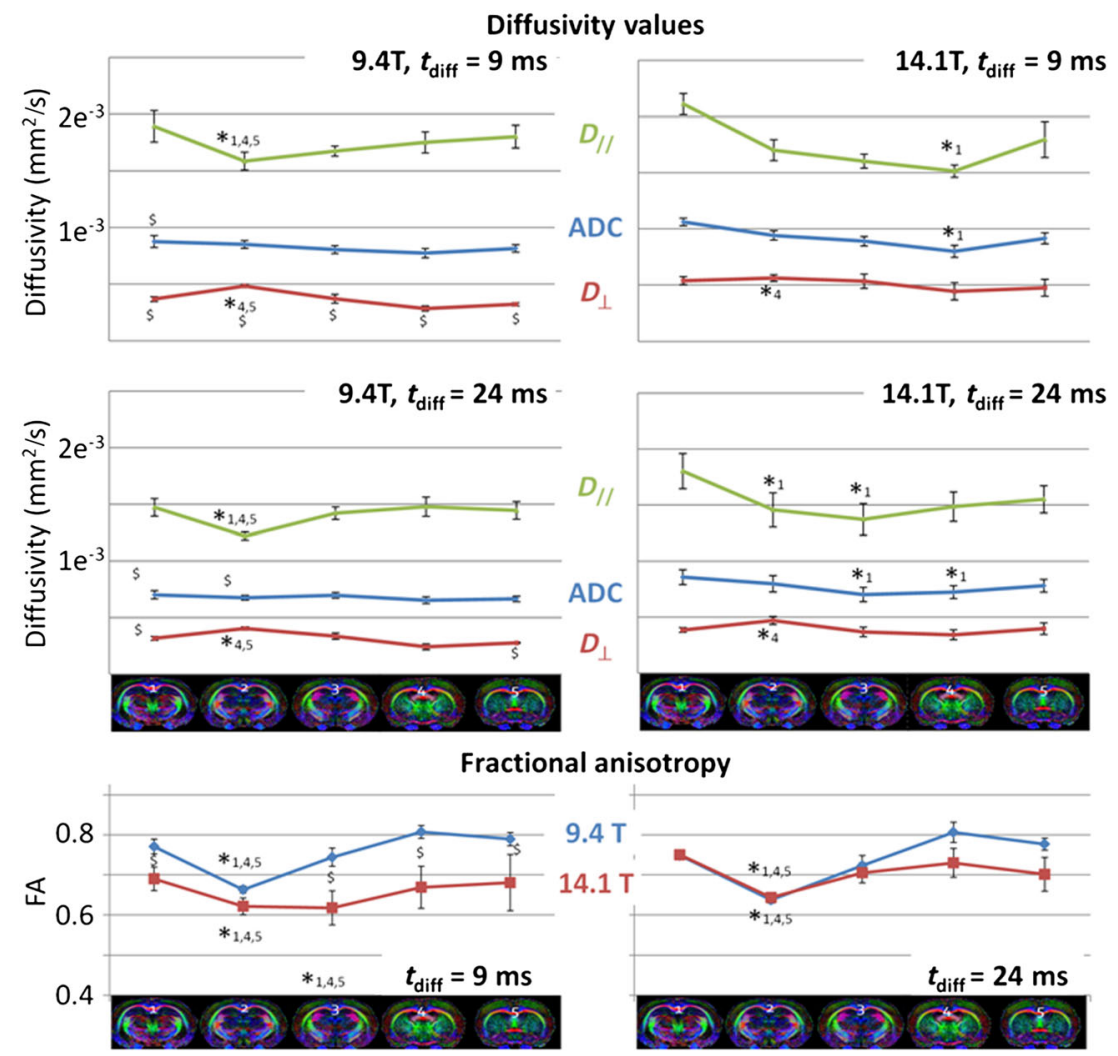

Figure 2. Plots of the mean diffusion tensor imaging (DTI)-derived parameters [apparent diffusion coefficient (ADC), axial diffusivity $\left(D_{/}\right)$, radial diffusivity $\left(D_{\perp}\right)$ and fractional anisotropy (FA) values \pm standard error of the mean (SEM)] measured in the corpus callosum as a function of the image plane at 9.4 and $14.1 \mathrm{~T}$ for the two extreme diffusion times ( $t_{\text {diff }}=9 \mathrm{~ms}$ and $t_{\text {diff }}=24 \mathrm{~ms}$ ) used in the study. $\left({ }^{*} p<0.05\right.$, Friedman test for a given $B_{0}$, indices correspond to the compared image planes, i.e. results of a multiple comparison of median cells, e.g. ${ }^{*} 1,4,5$, means that the current image plane is significantly different from image planes 1, 4 and 5; $\$ p<0.05$, Mann-Whitney test, 9.4 T versus 14.1 T.) An obvious regional difference in DTI-derived parameters was observed independent of the diffusion time and $B_{0}$. 
image plane 2 in Fig. 1 exhibited significantly higher $D_{\perp}$ and lower $D_{/ /}$values, which resulted in a lower FA than in image planes 1, 4 and/or 5, which corresponded to the splenium, the frontal part of the corpus callosum and the genu, respectively. This pattern of DTI-derived parameters along the corpus callosum was roughly the same for all measured $t_{\text {diff }}$ as well as both $B_{0}$ (Fig. 2).

\section{Diffusion time effect at $9.4 \mathrm{~T}$}

At 9.4T, there was no significant difference in FA values as a function of the diffusion time (Fig. 3). Several differences were observed in the diffusivity values between $t_{\text {diff }}=9 \mathrm{~ms}$ and longer diffusion times (see Fig. 3 for details).

\section{Diffusion time effect at $\mathbf{1 4 . 1} \mathrm{T}$}

At 14.1 T, FA values were found to be significantly lower for $t_{\text {diff }}=9 \mathrm{~ms}$ than for $t_{\text {diff }}=11-24 \mathrm{~ms}$, independently of the image plane. FA was also found to be significantly lower at $t_{\text {diff }}=11 \mathrm{~ms}$, although only at image planes 2 and 3 (Fig. 3). Smaller $D_{\perp}$ at long $t_{\text {diff }}$ was responsible for higher FA.

\subsection{T versus $14.1 \mathrm{~T}$}

At 14.1 T, FA was lower at shorter $t_{\text {diff }}$ and increased with $t_{\text {diff }}$ before a plateau was reached (Fig. 3), whereas FA values were constant at 9.4T. At 14.1 T, the diffusion time at which FA reached a plateau was also dependent on the axonal structure: it was longer in regions with the lowest FA, which corresponded to the body of the corpus callosum (image plane 3 in Fig. 3).
When comparing the results at 9.4 and 14.1 T, FA values were only significantly lower at $14.1 \mathrm{~T}$ relative to $9.4 \mathrm{~T}$ at the shortest $t_{\text {diff }}(9 \mathrm{~ms})$. At longer $t_{\text {diff }}(\geq 11 \mathrm{~ms})$, FA values were not found to be significantly different between 9.4 and 14.1 T. This low FA at 14.1 T for $t_{\text {diff }}=9 \mathrm{~ms}$ was predominantly caused by a larger $D_{\perp}$.

\section{$T_{2}$ measurements}

For the $T_{2}$ measurements, the global apparent $T_{2}$ values, obtained without the application of diffusion gradients, were $27.6 \pm 1.1 \mathrm{~ms}$ at $9.4 \mathrm{~T}\left(T_{2} 9.4 \mathrm{~T}\right)$ and $21.8 \pm 0.2 \mathrm{~ms}$ at $14.1 \mathrm{~T}$ $\left(T_{2} 14.1 \mathrm{~T}\right)$. With the diffusion gradients applied perpendicular to the main direction of the corpus callosum fibers, the apparent diffusion-weighted $T_{2}$ values in the corpus callosum (Fig. 4) were $26.0 \pm 1.2 \mathrm{~ms}$ at $9.4 \mathrm{~T}\left(T_{2} \mathrm{DW} 9.4 \mathrm{~T}\right)$ and $17.6 \pm 1.9 \mathrm{~ms}$ at $14.1 \mathrm{~T}$ $\left(T_{2} \mathrm{DW} 14.1 \mathrm{~T}\right)$. For both $B_{0}$, global apparent $T_{2}$ in the corpus callosum (Fig. 4) was significantly longer than the apparent diffusion-weighted $T_{2}(p<0.05)$.

\section{DISCUSSION}

Even though an increase in SNR is expected at higher $B_{0}$, the SNR in this study was not significantly different between 9.4 and 14.1 T. A short-TE spectroscopic SNR comparison has already been performed between our scanners, using as similar experimental set-ups as possible $(4,34)$, and only a small increase $(10-20 \%)$ has been observed at 14 T [for tentative reasons, see ref. (4)]. At longer TEs, this SNR increase is offset by the $T_{2}$ decrease at $14.1 \mathrm{~T}: 21.8 \mathrm{~ms}$ compared with $27.6 \mathrm{~ms}$ at 9.4 T. At TE $=50 \mathrm{~ms}$, this leads to a reduction in the relative signal intensity of $38 \%$. At lower magnetic field

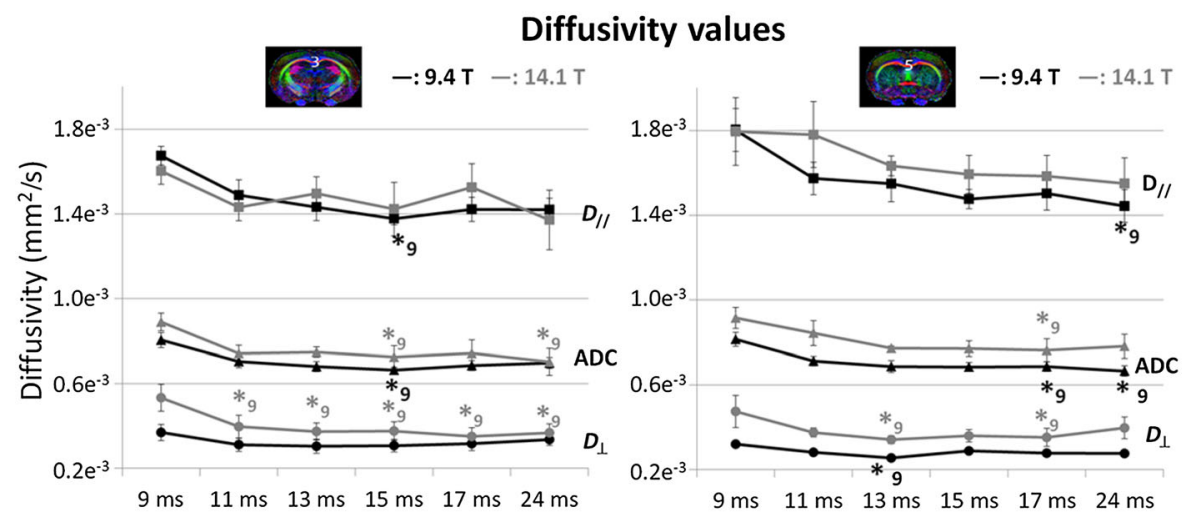

Fractional anisotropy

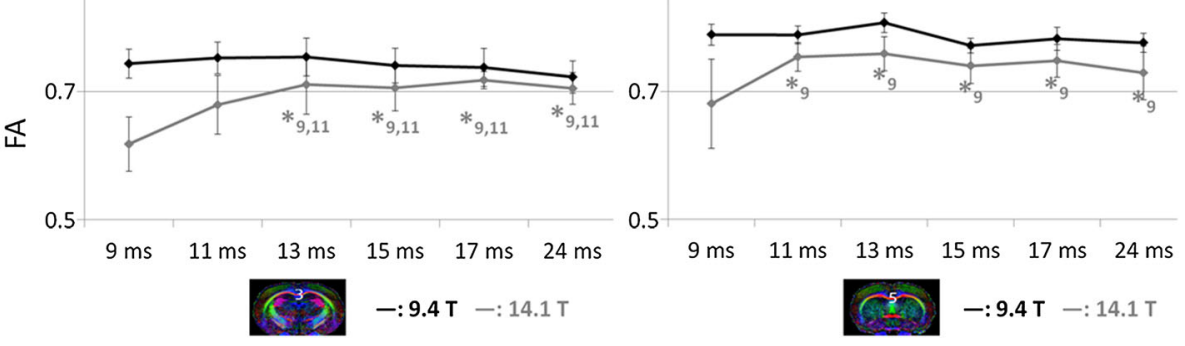

Figure 3. Plots of the mean diffusion tensor imaging (DTI)-derived parameters [apparent diffusion coefficient (ADC), axial diffusivity ( $\left.D_{/ /}\right)$, radial diffusivity $\left(D_{\perp}\right)$ and fractional anisotropy (FA) values \pm standard error of the mean (SEM)] as a function of $t_{\text {diff }}$ at $9.4 \mathrm{~T}$ (black) and $14.1 \mathrm{~T}$ (gray) for two different image planes of the rat brain corpus callosum, image plane 3 (left) and image plane 5 (right), which present different cellular structures. $\left[{ }^{*} p<0.05\right.$, Friedman test for a given $B_{0}(9.4 \mathrm{~T}$, black symbol; $14.1 \mathrm{~T}$, gray symbol), indices correspond to the compared diffusion times, i.e. results of a multiple comparison of median cells, e.g. ${ }^{*} 9,11$, means that the value for the current diffusion time is significantly different from the values obtained with $t_{\text {diff }}=9 \mathrm{~ms}$ and $11 \mathrm{~ms}$. 

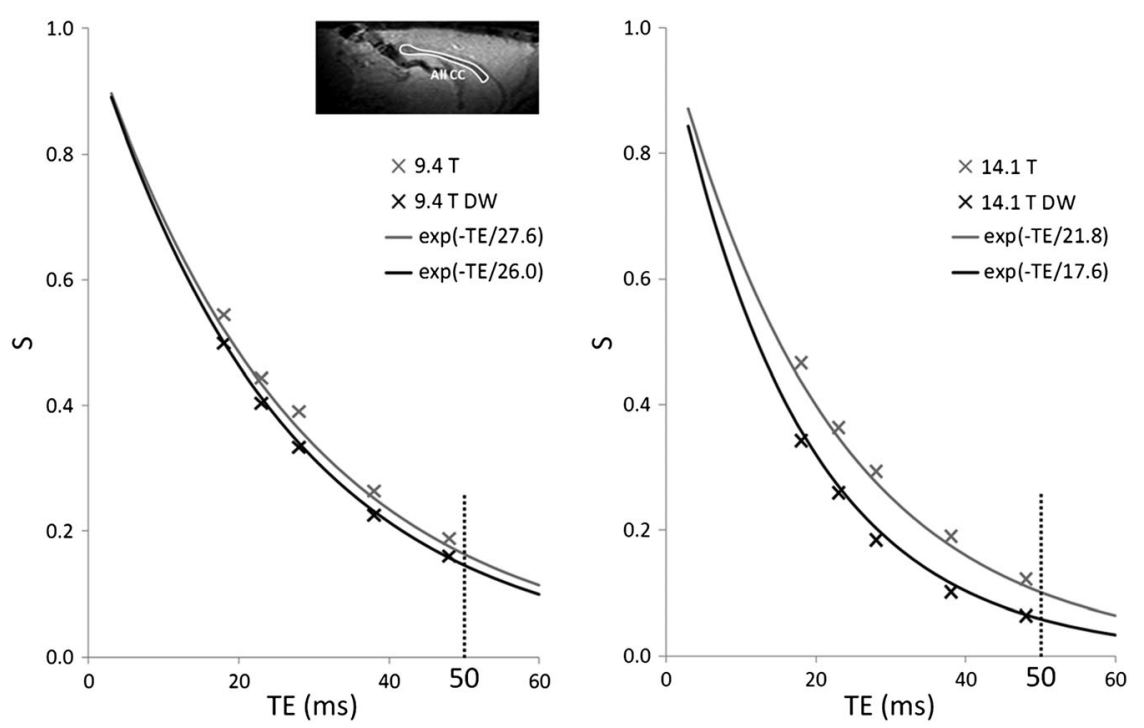

Figure 4. Inset: region of interest (ROI) of the corpus callosum (All CC) overlaid on a sagittal $T_{2}$-weighted image acquired with the stems sequence (TE/TM/TR $=18 / 11 / 3000 \mathrm{~ms}, b=0$ ) used for the $T_{2}$ measurements with and without diffusion weighting (DW). Scatter plots of the normalized signal intensities measured with (black crosses) and without (gray crosses) diffusion gradient for a typical rat at 9.4 T (left) and $14.1 \mathrm{~T}$ (right), as well as plots of $\exp \left(-\mathrm{TE} / T_{2}\right)$ for each mean $T_{2}$ value over the four rats derived from the fit with (black line) and without (gray line) the diffusion gradient at $9.4 \mathrm{~T}$ (left) and at $14.1 \mathrm{~T}$ (right).

strengths, Qin et al. (25) reported a gain in SNR from 1.5 to 3.0 T, even at long TE (i.e. $160 \mathrm{~ms}$ ). Nevertheless, a direct comparison is difficult as, from 1.5 to $3.0 \mathrm{~T}$, SNR shows an approximately linear increase with $B_{0}$ (35), whereas, with a TE of $160 \mathrm{~ms}$, the reduction in the relative signal caused by the $T_{2}$ decrease is only $30 \%$.

The direction-encoded color maps obtained were of high quality, with high SNR for both magnetic field strengths, leading to an accurate quantification of DTI-derived parameters (36).

The FA changes reported along the different slices demonstrated the well-known nonuniform axonal structure along the corpus callosum, as the axonal diameter changes throughout the corpus callosum in the antero-posterior direction, with a larger diameter in the central body, which contains motor axons, than in the splenium and genu (37). The increase in FA in regions with smaller axonal diameter is a result of two phenomena: $D_{\perp}$ decreases and $D_{/ /}$increases (Fig. 2). In these regions, a decrease in $D_{\perp}$ is expected because of the greater restriction of the mobility of molecules perpendicular to the mean direction. The $D_{/ /}$increase is more difficult to explain. It has been proposed that a small mean axonal diameter leads to a high axonal density, which increases $D_{/ /}$because of fewer diffusion disturbances along the fibers (37). This result shows the potential of probing microstructure by DTI-derived parameters. Independent of the imaging parameters $\left(t_{\text {diff }}\right.$ and/or $\left.B_{0}\right)$, the same pattern was observed along the rat corpus callosum.

In summary, two main differences were found between 9.4 and 14.1 T. First, at 9.4 T, FA values remained constant over the diffusion time, whereas, at 14.1 T, FA values increased with diffusion time before reaching a plateau. Second, the FA values at short $t_{\text {diff }}(9 \mathrm{~ms})$ were significantly lower at 14.1 than at 9.4 T. At longer $t_{\text {diff, }}$ this difference in FA values disappeared. However, the significance of these results may be limited by the small range of diffusion times assessed and may require more precise measurements, for instance by using oscillating diffusion gradients that would allow a lower $t_{\text {diff }}$ (38). A longer $t_{\text {diff }}$ would also provide important information and could be achieved using a stimulated echo pulse sequence.
The changes in the DTI-derived parameters as a function of $B_{0}$ reported in this study are in agreement with the recent results published by Polders et al. (26), who compared 1.5 and 7T. Nevertheless, several studies performed at lower field strength (1.5 $\mathrm{T}$ versus $3 \mathrm{~T}$ ) have shown contradictory results, with an FA increase with $B_{0}$ (23-25). Moreover, several studies have not reported any difference in FA values between 1.5 and $3 \mathrm{~T}$ (27) or between 3 and 7T (28). However, all of these studies, including the current work, tend to agree on a compartmentation of the signal weighted by different apparent $T_{2}$ relaxation times ('slow' and 'fast'). Thus, it is reasonable to assume that the contribution of each pool to the MR signal changes as a function of TE and $B_{0}$.

A simple two-pool model was used to illustrate our findings, which describes the white matter in a first hindered compartment (often associated with the extra-axonal space), characterized by a faster orthogonal diffusion and a lower FA, and a second restricted compartment (often associated with the intra-axonal space), characterized by a slower orthogonal diffusion (i.e. orthogonal to the axon direction) and a higher FA (13). A third pool, corresponding to water trapped in the myelin sheath, should also be considered, but it is assumed that the $T_{2}$ value of the myelin compartment is so small that its contribution is negligible in the range of TE values used in this study (39). The relative signal intensity of these two pools mostly depends on the diffusion acquisition parameters used. At very short diffusion time, water molecules do not have time to interact with the surrounding environment (e.g. membranes, organelles, etc.), and so both pools experience hindered diffusion and cannot be distinguished from one another. However, when increasing $t_{\text {diff, }}$ the mean displacement of water molecules also increases, making the diffusion-weighted signal more sensitive to restrictions of molecular motion. Therefore, depending on the $t_{\text {diff }}$ used, the compartment weighting of the diffusion signal can vary from a single hindered component to a mixture of hindered and restricted. As seen in the FA value (Fig. 3), the relative contribution of each compartment stabilizes once the mean water molecule displacement reaches the largest microcellular 
$f_{r}$ : Contribution to the

$$
\text { DW signal }
$$

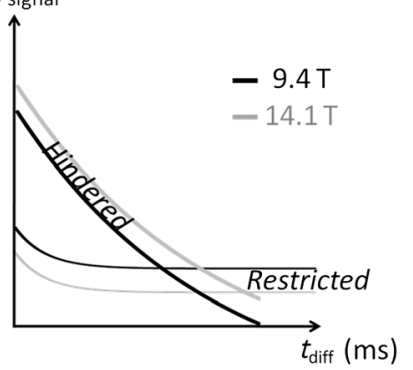

Figure 5. Schematic representation of the estimate of the fraction of the diffusion-weighted (DW) signal $\left(f_{\mathrm{r}}\right)$ as a function of the diffusion time $\left(t_{\text {diff }}\right)$ at $9.4 \mathrm{~T}$ (black lines) and $14.1 \mathrm{~T}$ (gray lines). At short $t_{\text {diff, }}$ the diffusion-weighted signal arises predominantly from the hindered compartment; at long $t_{\text {diff, }}$ the predominant contribution to the diffusionweighted signal arises from the restricted compartment.

structure scale. In addition to acquisition parameters, the diffusion-weighted signal may also be affected by different relaxation times between the two water pools, as well as by water exchange between the pools. However, the latter process can be assumed to be negligible at diffusion times around $9 \mathrm{~ms}$ according to the timescale of water exchange (40).

Differences between 9.4 and $14.1 \mathrm{~T}$ reported in this study originate from $B_{0}$-dependent parameters: system architecture differences, global SNR and/or relaxation times (longitudinal $T_{1}$ and transverse $T_{2}$ ). System differences (i.e. coil performances) influenced mostly the image quality (i.e. the global SNR), and SNR was similar at both $B_{0}$ values. With TE/TR $=50 / 2000 \mathrm{~ms}$, the differences in relative signal between 9.4 and $14.1 \mathrm{~T}$ caused by relaxation time differences were $8 \%$ and $60 \%$ for $T_{1}(5,30)$ and $T_{2}$, respectively. Moreover, a $T_{2}$ effect on DTI-derived parameters has already been mentioned in several studies (23-25).

To assess this potential $T_{2}$ effect, $T_{2}$ measurements were performed with and without the application of diffusion gradients: without the diffusion gradient, about $70-80 \%$ of the signal was assumed to arise from the hindered pool and it decayed with a global apparent $T_{2}$, whereas, with diffusion gradients applied perpendicular to the fiber direction of the corpus callosum, the signal was presumed to be predominantly restricted, leading to a pseudo- $T_{2}$ estimate of the restricted pool. We found a significantly lower $T_{2}$ for the restricted compartment relative to the global $T_{2}$ at both $B_{0}$ values. More interestingly, at TE $=50 \mathrm{~ms}$, the decreases in $\exp \left(-\mathrm{TE} / T_{2}\right)$ between the two pools were $11 \%$ and $41 \%$ at $9.4 \mathrm{~T}$ and $14.1 \mathrm{~T}$, respectively (Fig. 4). This suggests that the signal contribution of the restricted compartment is smaller at 14.1 T at the TE used in this study, and may explain the lower FA reported at short diffusion times. Furthermore, although, at low $B_{0}$, TE is usually shorter than the $T_{2}$ of the slow pool, at ultrahigh magnetic field, the TE used is, most of the time, longer (e.g. about twice in the current study) than the $T_{2}$ of both pools. As such, the signal weighting effects are increased at high $B_{0}$, which may explain the contradictory results reported at lower magnetic fields (23-25).

This result is supported by the Bloembergen-Purcell-Pound relaxation theory, in which the transverse spin-spin relaxation $T_{2}$ is a well-known tissue-specific time constant that characterizes the loss of coherent transverse magnetization over time, and is lower in a more solid environment. One can imagine that, in a more confined (restricted) environment with numerous interactions, energy exchange would be larger and the environment would thus be characterized by a shorter T2 value (when compared with hindered), which is in good agreement with the results of the current study. Indeed, in a study on frog sciatic nerves, Peled et al. (41) suggested that spins that are extracellular and unbounded have a longer $T_{2}$ than those located in intracellular compartments.

The differences in the FA values as a function of $t_{\text {diff }}$ between the two $B_{0}$ values can also be explained by this difference in $T_{2}$ : as the amount of species with short $T_{2}$ is lower at $14.1 \mathrm{~T}$, the predominance of the restricted compartment is reached at longer $t_{\text {diff, }}$ whereas, at $9.4 \mathrm{~T}$, even at $t_{\text {diff }}=9 \mathrm{~ms}$, the diffusionweighted signal arises predominantly from the restricted compartment (Fig. 5). In addition, in regions with higher axonal diameter (image planes 2 and 3), this characteristic diffusion time tends to be even longer, most probably as a result of the different cellular structures present.

At such high magnetic field strength, it is important to note that the shorter diffusion-weighted $T_{2}$ relaxation time constant may be driven by diffusion through susceptibility-induced local magnetic field gradients (1). This being the case, the differences in DTIderived parameters found at $14.1 \mathrm{~T}$ could be related to a relative loss of signal from spins close to susceptibility barriers, which also serve as diffusion barriers, at the relatively long TEs used for this study. Thus, signal from the spins whose displacements are mostly hindered by barriers may be less 'visible' at higher field strength. Even though this compartmentation of the diffusion-weighted signal has been shown recently in several publications [ref. (11) and references cited therein], the exact nature of the compartments will require further experiments to be fully understood.

In conclusion, this study reports differences in the DTI-derived parameters as a function of the diffusion time, as well as of the magnetic field strength. Although the changes as a function of the diffusion time have already been reported $(15,20,38,42)$, and can be explained by the diffusion theory (i.e. for each diffusion time, different structural scales are probed), the changes with $B_{0}$ remain more difficult to explain. Even so, our results provide evidence for an MR signal compartmentation, corroborating with recent developments in diffusion theory, such as the composite hindered and restricted model of diffusion (CHARMED) (13). This study suggests that the observed differences in DTI-derived parameters between the two $B_{0}$ values may be related to differences in apparent $T_{2}$ over the pools. Nevertheless, there is no evidence to distinguish a spatial compartment (e.g. intra- or extra-cellular) from a water buffer around the susceptibility barriers. Further investigation will be necessary to solve this problem, for example multi-component $T_{2}$ measurements combined with more advanced diffusion modeling and reconstruction [ref. (11) and references cited therein]. We conclude that it is likely that a field-dependent differential reduction in $T_{2}$ relaxation times could result in fielddependent differences in DTI-derived parameters.

\section{Acknowledgements}

This work was supported by the Swiss National Fund $N^{\circ} 31003 \mathrm{~A}-$ 112233, FP7 European project: CONNECT, the Centre d'Imagerie Biomédicale (CIBM) of the Université de Genève (UNIGE), Université de Lausanne (UNIL), Hôpitaux Universitaires de Genève (HUG), Centre Hospitalier Universitaire Vaudois Lausanne (CHUV), Ecole Polytechnique Fédérale de Lausanne (EPFL) and the Leenards and Jeantet Foundations. The authors thank Dr Kieran O'Brien, Dr Vladimir Mlynarik and Dr Ruud van Heeswijk for their help in revising the manuscript. 


\section{REFERENCES}

1. Callaghan PT. Principles of Nuclear Magnetic Resonance Microscopy. Oxford University Press: New York; 1991.

2. Marques JP, Maddage R, Mlynarik V, Gruetter R. On the origin of the MR image phase contrast: an in vivo MR microscopy study of the rat brain at 14.1 T. Neuroimage 2009; 46(2): 345-352.

3. Mekle R, Mlynarik V, Gambarota G, Hergt M, Krueger G, Gruetter R. MR spectroscopy of the human brain with enhanced signal intensity at ultrashort echo times on a clinical platform at $3 \mathrm{~T}$ and $7 \mathrm{~T}$. Magn. Reson. Med. 2009; 61(6): 1279-1285.

4. Mlynarik V, Cudalbu C, Xin L, Gruetter R. ${ }^{1} \mathrm{H}$ NMR spectroscopy of rat brain in vivo at 14.1 Tesla: improvements in quantification of the neurochemical profile. J. Magn. Reson. 2008; 194(2): 163-168.

5. de Graaf RA, Brown PB, Mclntyre S, Nixon TW, Behar KL, Rothman DL. High magnetic field water and metabolite proton $\mathrm{T} 1$ and $\mathrm{T} 2$ relaxation in rat brain in vivo. Magn. Reson. Med. 2006; 56(2): 386-394.

6. Basser PJ, Mattiello J, LeBihan D. MR diffusion tensor spectroscopy and imaging. Biophys. J. 1994; 66(1): 259-267.

7. Cha S. Neuroimaging in neuro-oncology. Neurotherapeutics 2009; 6(3): 465-477.

8. Sizonenko SV, Camm EJ, Garbow JR, Maier SE, Inder TE, Williams CE, Neil JJ, Huppi PS. Developmental changes and injury induced disruption of the radial organization of the cortex in the immature rat brain revealed by in vivo diffusion tensor MRI. Cereb. Cortex 2007; 17(11): 2609-2617.

9. van de Looij Y, Mauconduit F, Beaumont M, Valable S, Farion R, Francony G, Payen J-F, Lahrech H. Diffusion tensor imaging of diffuse axonal injury in a rat brain trauma model. NMR Biomed. 2012; 25(1): 93-103.

10. Zhang J, van Zijl PC, Laterra J, Salhotra A, Lal B, Mori S, Zhou J. Unique patterns of diffusion directionality in rat brain tumors revealed by high-resolution diffusion tensor MRI. Magn. Reson. Med. 2007; 58(3): 454-462.

11. Yablonskiy DA, Sukstanskii AL. Theoretical models of the diffusion weighted MR signal. NMR Biomed. 2011; 23(7): 661-681.

12. Alexander DC, Hubbard PL, Hall MG, Moore EA, Ptito M, Parker GJ, Dyrby TB. Orientationally invariant indices of axon diameter and density from diffusion MRI. Neuroimage 2010; 52(4): 1374-1389.

13. Assaf Y, Freidlin RZ, Rohde GK, Basser PJ. New modeling and experimental framework to characterize hindered and restricted water diffusion in brain white matter. Magn. Reson. Med. 2004; 52(5): 965-978.

14. Clark CA, Le Bihan D. Water diffusion compartmentation and anisotropy at high b values in the human brain. Magn. Reson. Med. 2000; 44(6): 852-859.

15. Stanisz GJ, Szafer A, Wright GA, Henkelman RM. An analytical model of restricted diffusion in bovine optic nerve. Magn. Reson. Med. 1997; 37(1): 103-111.

16. Zhang H, Schneider T, Wheeler-Kingshott CA, Alexander DC. NODDI: practical in vivo neurite orientation dispersion and density imaging of the human brain. Neuroimage, 2012; 61(4): 1000-1016.

17. MacKay A, Laule C, Vavasour I, Bjarnason T, Kolind S, Madler B. Insights into brain microstructure from the T2 distribution. Magn. Reson. Imaging, 2006; 24(4): 515-525.

18. MacKay A, Whittall K, Adler J, Li D, Paty D, Graeb D. In vivo visualization of myelin water in brain by magnetic resonance. Magn. Reson. Med. 1994; 31(6): 673-677.

19. Buckley DL, Bui JD, Phillips MI, Zelles T, Inglis BA, Plant HD, Blackband SJ. The effect of ouabain on water diffusion in the rat hippocampal slice measured by high resolution NMR imaging. Magn. Reson. Med. 1999; 41(1): 137-142.

20. Harkins KD, Galons JP, Secomb TW, Trouard TP. Assessment of the effects of cellular tissue properties on ADC measurements by numerical simulation of water diffusion. Magn. Reson. Med. 2009; 62(6): 1414-1422.

21. Schoeniger JS, Aiken N, Hsu E, Blackband SJ. Relaxation-time and diffusion NMR microscopy of single neurons. J. Magn. Reson. 1994; 103(3): 261-273.
22. Vestergaard-Poulsen $P$, Hansen $B$, Ostergaard $L$, Jakobsen $R$. Microstructural changes in ischemic cortical gray matter predicted by a model of diffusion-weighted MRI. J. Magn. Reson. Imaging, 2007; 26(3): 529-540.

23. Fushimi $Y$, Miki $Y$, Okada T, Yamamoto A, Mori N, Hanakawa T, Urayama S, Aso T, Fukuyama H, Kikuta K, Togashi K. Fractional anisotropy and mean diffusivity: comparison between 3.0-T and 1.5-T diffusion tensor imaging with parallel imaging using histogram and region of interest analysis. NMR Biomed. 2007; 20(8): 743-748.

24. Huisman TA, Loenneker T, Barta G, Bellemann ME, Hennig J, Fischer JE, II'yasov KA. Quantitative diffusion tensor MR imaging of the brain: field strength related variance of apparent diffusion coefficient (ADC) and fractional anisotropy (FA) scalars. Eur. Radiol. 2006; 16(8): 1651-1658.

25. Qin W, Yu CS, Zhang F, Du XY, Jiang H, Yan YX, Li KC. Effects of echo time on diffusion quantification of brain white matter at $1.5 \mathrm{~T}$ and 3.0 T. Magn. Reson. Med. 2009; 61(4): 755-760.

26. Polders DL, Leemans A, Hendrikse J, Donahue MJ, Luijten PR, Hoogduin JM. Signal to noise ratio and uncertainty in diffusion tensor imaging at 1.5, 3.0, and 7.0 Tesla. J. Magn. Reson. Imaging, 2011; 33(6): 1456-1463.

27. Alexander AL, Lee JE, Wu YC, Field AS. Comparison of diffusion tensor imaging measurements at 3.0 T versus $1.5 \mathrm{~T}$ with and without parallel imaging. Neuroimag. Clin. N. Am. 2006; 16(2): 299-309, xi.

28. Choi S, Cunningham DT, Aguila F, Corrigan JD, Bogner J, Mysiw WJ, Knopp MV, Schmalbrock P. DTI at 7 and 3 T: systematic comparison of SNR and its influence on quantitative metrics. Magn. Reson. Imaging, 2011; 29(6): 739-751.

29. Gruetter R, Tkac I. Field mapping without reference scan using asymmetric echo-planar techniques. Magn. Reson. Med. 2000; 43(2): 319-323.

30. van de Looij Y, Kunz N, Huppi P, Gruetter R, Sizonenko S. Diffusion tensor echo planar imaging using surface coil transceiver with a semiadiabatic RF pulse sequence at $14.1 \mathrm{~T}$. Magn. Reson. Med. 2011; 65(3): 732-737.

31. Hasan KM, Parker DL, Alexander AL. Comparison of gradient encoding schemes for diffusion-tensor MRI. J. Magn. Reson. Imaging, 2001; 13(5): 769-780.

32. Conturo TE, McKinstry RC, Aronovitz JA, Neil JJ. Diffusion MRI: precision, accuracy and flow effects. NMR Biomed. 1995; 8(7-8): 307-332.

33. Frahm J, Merboldt KD, Hänicke W, Haase A. Stimulated echo imaging. J. Magn. Reson. 1985; 64(1): 81-93.

34. van Heeswijk RB, Pilloud Y, Morgenthaler FD, Gruetter R. A comparison of in vivo ${ }^{13} \mathrm{C}$ MR brain glycogen quantification at 9.4 and $14.1 \mathrm{~T}$. Magn. Reson. Med. 2012; 67(6): 1523-1527.

35. Norris DG. High field human imaging. J. Magn. Reson. Imaging 2003; 18(5): 519-529.

36. Hunsche $S$, Moseley ME, Stoeter $P$, Hedehus M. Diffusion-tensor MR imaging at 1.5 and 3.0 T: initial observations. Radiology, 2001; 221(2): 550-556.

37. Barazany D, Basser PJ, Assaf Y. In vivo measurement of axon diameter distribution in the corpus callosum of rat brain. Brain 2009; 132(Pt 5): 1210-1220.

38. Schachter M, Does MD, Anderson AW, Gore JC. Measurements of restricted diffusion using an oscillating gradient spin-echo sequence. J. Magn. Reson. 2000; 147(2): 232-237.

39. Whittall KP, MacKay AL, Graeb DA, Nugent RA, Li DK, Paty DW. In vivo measurement of $\mathrm{T} 2$ distributions and water contents in normal human brain. Magn. Reson. Med. 1997; 37(1): 34-43.

40. van der Toorn A, Sykova E, Dijkhuizen RM, Vorisek I, Vargova L, Skobisova $E$, van Lookeren Campagne $M$, Reese $T$, Nicolay $K$. Dynamic changes in water ADC, energy metabolism, extracellular space volume, and tortuosity in neonatal rat brain during global ischemia. Magn. Reson. Med. 1996; 36(1): 52-60.

41. Peled S, Cory DG, Raymond SA, Kirschner DA, Jolesz FA. Water diffusion, $\mathrm{T}(2)$, and compartmentation in frog sciatic nerve. Magn. Reson. Med. 1999; 42(5): 911-918.

42. Clark CA, Hedehus M, Moseley ME. Diffusion time dependence of the apparent diffusion tensor in healthy human brain and white matter disease. Magn. Reson. Med. 2001; 45(6): 1126-1129. 